\section{A tomografia por emissão de pósitrons: uma nova modalidade na medicina nuclear brasileira}

\section{Cecil Chow Robilotta ${ }^{1}$}

Como citar: Robilotta CC. A tomografia por emissão de pósitrons: uma nova modalidade na medicina nuclear brasileira. Rev Panam Salud Publica. 2006; 20(2/3):134-42.

Palavras-chave: compostos radiofarmacêuticos, diagnóstico por imagem, PET, imagem funcional, Brasil.

\footnotetext{
1 Universidade de São Paulo, Instituto de Física. Correspondência: Rua do Matão, Travessa R, 187, Cidade Universitária, Butantã, CEP 05508-900, São Paulo, SP, Brasil. E-mail: cecilcr@if.usp.br
}

\section{SINOPSE}

A medicina nuclear utiliza substâncias radioativas para diagnosticar e tratar doenças. Essa especialidade médica, capaz de fornecer informações fisiológicas e metabólicas sobre o corpo humano, se tornou uma ferramenta fundamental para a detecção precoce de muitas desordens, inclusive vários tipos de câncer. O presente artigo descreve os marcos históricos da medicina nuclear, os princípios físicos básicos que subjazem à tomografia por emissão de pósitrons (PET), um método de imagem usado para mapear a distribuição de radiofármacos no corpo para fins diagnósticos e terapêuticos, $e$ o estado atual dessa modalidade no Brasil.

A medicina nuclear é uma especialidade médica que utiliza compostos (ou moléculas) marcados com radionuclídeos, os radiofármacos, para fins de diagnóstico e terapia. Esses compostos seguem caminhos funcionais ou metabólicos específicos dentro dos pacientes, o que confere a essa modalidade diagnóstica uma característica de natureza biológica que as outras modalidades não possuem. A detecção externa da radiação emitida pelo radiofármaco permite diagnosticar precocemente muitas doenças, enquanto que as alterações anatômicas, muitas vezes, não se manifestam senão em estágios relativamente avançados, como no caso de diversos tipos de câncer.

Outra característica importante dos exames realizados com radiofármacos é a sua alta sensibilidade-isto é, é possível obter informações biológicas com concentrações de radiofármacos em níveis de nano ou picomolares. Além disso, a marcação de diferentes moléculas com um único radionuclídeo permite avaliações e estudos de um mesmo órgão ou sistema em seus aspectos tanto macroscópicos quanto moleculares. Tais estudos podem ser realizados através de imagens obtidas in vivo ou através de ensaios laboratoriais. Atualmente, a maior parte dos estudos radionuclídicos clínicos é de imagens, em especial as tomográficas.

Neste artigo, serão relatados, inicialmente, alguns fatos históricos sobre o desenvolvimento da medicina nuclear, seguidos de uma apresentação dos aspectos físicos da tomografia por emissão de pósitrons (positron emission tomography, PET); finalmente, será abordada a situação desta modalidade no Brasil. 


\section{UM POUCO DE HISTÓRIA}

Pode-se dizer que a história da medicina nuclear começou com as descobertas da radioatividade natural por Henri Becquerel, em 1896, e de elementos radioativos naturais por Marie e Pierre Curie, em 1898 (descobertas pelas quais os três cientistas receberam o Prêmio Nobel de Física de 1903). Entretanto, foi o "princípio do traçador", proposto pelo químico húngaro George de Hevesy (1), em 1913, que realmente forneceu o fundamento biológico para a especialidade. Ele confirmou o princípio através de experiências com nitrato de chumbo marcado com o nuclídeo radioativo ${ }^{210} \mathrm{~Pb}$, mostrando sua absorção e seu movimento em plantas. Por esse feito, Hevesy recebeu o Prêmio Nobel de Química de 1943.

Em 1927, Herrmann L. Blumgart e Soma Weiss (2) realizaram a primeira medida da velocidade sangüínea, mediante a injeção de uma solução de radônio-C em um braço e a subseqüente verificação, com uma câmara de Wilson, de sua chegada no outro braço. Um avanço significativo na quantificação de substâncias como os hormônios no sangue foi alcançado com a técnica de ensaios radioimunológicos (radioimmunoassay, RIA), desenvolvida por Solomon A. Berson e Rosalyn S. Yalow (3). Por esse trabalho, Yalow foi a primeira física a receber um Prêmio Nobel de Medicina e Fisiologia, em 1977.

Em 1932, a invenção e a construção do cíclotron, por Ernest O. Lawrence e M. Stanley Livingstone (4), possibilitaram a produção de radionuclídeos artificiais, através do bombardeamento de núcleos-alvos por partículas positivas aceleradas (Prêmio Nobel de Física para Lawrence em 1939). Entretanto, a produção de quantidades suficientes de radionuclídeos para uso médico só se iniciou com o advento dos reatores nucleares, desenvolvidos durante a Segunda Guerra Mundial. O reator de Oak Ridge (Estados Unidos) começou sua produção em escala comercial em 1946, e o de Harwell (Reino Unido), em 1947.

Inicialmente, havia poucos radionuclídeos adequados para as aplicações médicas, e grande parte dos estudos clínicos enfocava a avaliação da glândula tireóide e suas disfunções, com o uso do ${ }^{131}$ I na forma de iodeto. O principal detector usado era o contador Geiger-Müller, que indicava e media a presença do radiofármaco, sem, contudo, distinguir a energia da radiação gama detectada; tampouco produzia imagens da distribuição do composto.

Foi Benedict Cassen (5) quem, em 1951, ao inventar e construir o mapeador linear, deu início à era de diagnóstico por imagens radionuclídicas. Em 1958, Hal Anger (6) desenvolveu a câmara de cintilação, um sistema de formação de imagens que não exigia que o detector fosse movimentado e que apre- sentava maior resolução geométrica, além da possibilidade de se obter projeções diferentes de uma mesma distribuição de radiofármaco. As informações adquiridas pela câmara de cintilação eram transformadas em imagens e exibidas por um tubo de raios catódicos, de modo que podiam ser registradas em filmes ou chapas fotográficas. As modernas câmaras usadas hoje são derivadas da câmara Anger.

O grande poder diagnóstico da medicina nuclear se firmou quando Paul Harper (7) e sua equipe introduziram o radionuclídeo ${ }^{99 \mathrm{~m}} \mathrm{Tc}$ como marcador. Esse nuclídeo decai por transição isomérica, emite um fóton com energia de $140 \mathrm{keV}$, bastante adequado para a câmara que Anger inventou, e possui meia-vida física de 6 horas, possibilitando estudos em intervalos de tempo razoáveis. Além disso, ele é produzido pelo gerador ${ }^{99} \mathrm{Mo}-{ }^{99 \mathrm{~m}} \mathrm{Tc}$, um sistema que contém o par de radionuclídeos pai $\left({ }^{99} \mathrm{Mo}\right)$ - filho $\left({ }^{99 \mathrm{~m}} \mathrm{Tc}\right)$ e que permite a separação e a extração do elemento filho. O radionuclídeo ${ }^{99 \mathrm{~m}} \mathrm{Tc}$ é continuamente produzido pela desintegração do ${ }^{99} \mathrm{Mo}$, e sua extração periódica possibilita um fornecimento constante nos próprios centros de medicina nuclear (8). Outra característica muito importante é a facilidade com que o ${ }^{99 \mathrm{~m}} \mathrm{Tc}$ consegue marcar um número muito grande de fármacos, o que o torna aplicável em estudos de quase todos os órgãos e sistemas do corpo humano. Dados recentes da Sociedade de Medicina Nuclear dos Estados Unidos indicam que existem mais de 100 procedimentos diferentes na medicina nuclear para fins diagnósticos que utilizam radiofármacos específicos, que cobrem um número considerável de estudos sobre a fisiologia dos sistemas orgânicos do corpo (9).

Com o desenvolvimento dos computadores, nos anos 1960, foi possível adquirir, armazenar e processar as imagens obtidas com as câmaras de cintilação para, por exemplo, extrair parâmetros fisiológicos, corrigir distorções associadas ao processo de formação de imagens, assim como evidenciar estruturas de interesse. Na década de 1970, novos avanços em computação e, principalmente, no desenvolvimento e na implementação de métodos de reconstrução permitiram a realização de tomografias por emissão de fótons únicos (single photon emission computed tomography, SPECT —o que foi feito por David E. Kuhl e sua equipe na Universidade da Pensilvânia (10) — e de PET, por Gordon L. Brownell e colaboradores no Hospital Geral de Massachusetts $(11,12)$ e por Michael E. Phelps e colegas na Universidade da Califórnia em Los Angeles $(13,14)$. Vale destacar a contribuição de David Chesler (15) ao propor e reconstruir cortes tomográficos de emissão e transmissão pelo método da retroprojeção, em 1971. Variantes desse método de reconstrução ainda são muito usadas na rotina clínica. 
Uma parte dos procedimentos clínicos disponíveis utiliza as projeções planas das distribuições volumétricas contendo o radiofármaco para extrair informações, enquanto que a outra faz uso das imagens tomográficas por emissão, reconstruídas a partir das projeções, para apresentar os conteúdos em cortes ou volumes.

Os estudos realizados diretamente sobre as projeções podem ser estáticos ou dinâmicos. Estes são constituídos por séries temporais de imagens que acompanham determinado processo biológico. Pelo fato de as imagens serem projeções planas, não é possível a localização precisa do radiofármaco no corpo. No entanto, a presença e a evolução temporal desse material no sistema funcional específico são informações essenciais e, não raro, suficientes para a detecção de muitas moléstias que não podem ser detectadas por outros métodos de diagnóstico por imagens. Além disso, a possibilidade de quantificar parâmetros fisiológicos com processamentos adequados torna os estudos planos particularmente úteis, por exemplo, na avaliação das funções renais e do sistema gástrico.

Desde a sua introdução em aplicações clínicas, as técnicas de tomografia por emissão, SPECT e PET, vêm suprindo a comunidade médica com informações biológicas distribuídas no espaço e no tempo. Entretanto, devido à meia-vida física extremamente curta dos emissores de pósitron viáveis e ao alto custo de implantação e execução, só nos anos 1990 a tecnologia PET se fixou definitivamente, mesmo nos países desenvolvidos, na rotina de grande parte das clínicas nucleares, com o uso da ${ }^{18}$ F-fluordeoxiglicose (FDG), composto análogo à glicose, marcado inicialmente com ${ }^{14} \mathrm{C}$ por Louis Sokoloff e equipe (16) e, posteriormente, com ${ }^{18} \mathrm{~F}$ por Tatuo Ido e colaboradores (17). A SPECT, por outro lado, foi absorvida de imediato, e muitos radiofármacos (a maior parte marcada com ${ }^{99} \mathrm{~m} \mathrm{Tc}$ ) e procedimentos foram e continuam sendo desenvolvidos.

Associada ao desenvolvimento farmacológico, a pesquisa para melhorar a instrumentação, com o uso de detectores mais eficientes e de eletrônica mais rápida, tem impulsionado tanto a SPECT como a PET em suas aplicações. Em especial, a recente combinação da PET com a tomografia computadorizada (computed tomography, CT) - o sistema combinado PET/CT - por David W. Townsend e equipe, na Universidade de Pittsburgh (18), acoplando um tomógrafo por emissão de pósitrons a um outro multicortes por transmissão de raios $X$, permite a extração máxima dos benefícios que essas modalidades podem oferecer, em conjunto, aos médicos e seus pacientes.

Como conseqüência da evolução instrumental e farmacológica, as imagens radionuclídicas estão fornecendo informações cada vez mais em nível molecular, de modo que a escolha dos métodos de reconstrução tomográfica e as correções, assim como as quantificações em tomografia por emissão, têm merecido atenção especial por parte da comunidade. Pesquisas que enfocam a busca de resultados quantitativos mais precisos, confiáveis e rápidos estão sendo realizadas em inúmeros centros do mundo, inclusive no Brasil.

Segundo dados de 2002 da Comissão Nacional de Energia Nuclear (CNEN), existem, no território brasileiro, mais de 250 clínicas de medicina nuclear, com um número similar de câmaras SPECT, das quais cerca de $75 \%$ estão localizadas nas regiões Sudeste e Sul. Em 1998, entrou em operação, na Cidade de São Paulo, o primeiro sistema capaz de produzir imagens tomográficas com o uso de emissores de pósitron. O equipamento, uma câmara PET/ SPECT, podia também ser usado para a obtenção de imagens SPECT. Atualmente, além da Cidade de São Paulo, o Rio de Janeiro e Brasília também possuem clínicas que oferecem estudos de PET.

\section{TOMOGRAFIA POR EMISSÃO DE PÓSITRONS: ASPECTOS FÍSICOS}

A tomografia por emissão de pósitrons, ou PET, como o próprio nome diz, é um mapa da distribuição de um radiofármaco emissor de pósitrons em um determinado corte do corpo.

O decaimento por emissão de pósitron pode ser descrito por,

$$
{ }_{Z}^{A} X \rightarrow{ }_{Z-1}^{A} Y+{ }_{+1}^{0} e+v,
$$

onde o radionuclídeo pai ${ }_{Z}^{A} X$ decai para o nuclídeo filho ${ }_{Z-1}^{A} Y$, com a subseqüente emissão de um pósitron $\left(\beta^{+}\right)$e de um neutrino $(v)$.

A figura 1 ilustra o esquema de decaimento do ${ }_{6}^{11} C$ para ${ }_{5}^{11} B$, o caminho percorrido até a aniquilação do pósitron com um elétron do meio e a conseqüente formação do par de fótons de $511 \mathrm{keV}$ cada, em direções opostas. Esses fótons são detectados externamente, e a informação é usada para a reconstrução das tomografias.

A idéia de utilizar emissores de pósitrons para detectar tumores de cérebro foi proposta, em 1951, separadamente, por Gordon L. Brownell ao neurocirurgião William H. Sweet (19), e por Frank R. Wrenn e colaboradores (20). Entretanto, somente o sistema idealizado por Brownell (21) era capaz de produzir um mapa aproximado da distribuição do radiofármaco através da detecção do par de fótons de aniquilação com dois cristais de iodeto de sódio ativado com 
FIGURA 1. Tomografia por emissão de pósitrons (PET): esquema de decaimento do ${ }_{6}^{11} C$ para ${ }_{5}^{11} B$ e da aniquilação do pósitron com elétron e formação do par de fótons de $511 \mathrm{keV}$ cada, em direções opostas ${ }^{\mathrm{a}}$

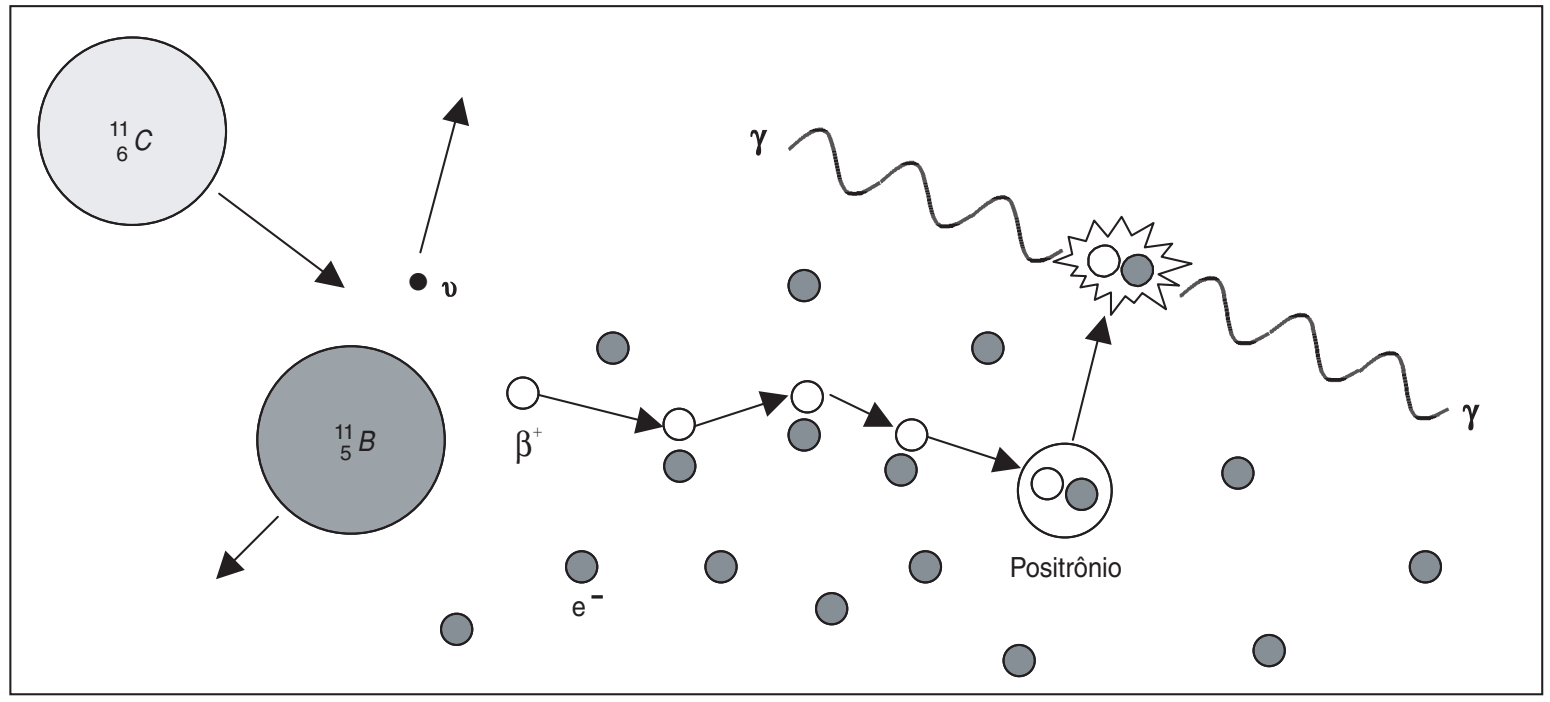

a $O$ positrônio é o sistema formado pelo pósitron e o elétron antes de sua aniquilação, e que resulta na produção do par de fótons.

TABELA 1. Principais emissores de pósitrons e suas características

\begin{tabular}{|c|c|c|c|}
\hline Radionuclídeo & $\begin{array}{l}\mathrm{T}_{1 / 2} \\
(\mathrm{~min})\end{array}$ & $\begin{array}{c}\mathrm{E}_{\beta_{+}} \max \\
(\mathrm{MeV})\end{array}$ & $\begin{array}{c}\text { Alcance máximo } \\
\text { em água }(\mathrm{mm})\end{array}$ \\
\hline \multicolumn{4}{|l|}{ Carbono-11 } \\
\hline${ }^{11} \mathrm{C}$ & 20,4 & 0,959 & 5,0 \\
\hline \multicolumn{4}{|l|}{ Nitrogênio-13 } \\
\hline${ }^{13} \mathrm{~N}$ & 9,96 & 1,197 & 5,4 \\
\hline \multicolumn{4}{|l|}{ Oxigênio-15 } \\
\hline${ }^{15} \mathrm{O}$ & 2,07 & 1,738 & 8,2 \\
\hline \multicolumn{4}{|l|}{ Flúor-18 } \\
\hline${ }^{18} \mathrm{~F}$ & 109,8 & 0,650 & 2,4 \\
\hline \multicolumn{4}{|l|}{ Gálio-68 } \\
\hline${ }^{68} \mathrm{Ga}$ & 68 & 1,899 & 9,4 \\
\hline \multicolumn{4}{|l|}{ Rubídio-82 } \\
\hline${ }^{82} \mathrm{Rb}$ & 1,3 & 3,350 & 15,6 \\
\hline
\end{tabular}

tálio $[\mathrm{NaI}(\mathrm{Tl})]$, colocados em lados opostos da cabeça do paciente e acoplados a um sistema de varredura (22). Ainda nos anos 1950, Michel M. Ter-Pogossian e William E. Powers (23) determinaram o conteúdo de oxigênio em neoplasias malignas com ${ }^{15} \mathrm{O}$.

Os radionuclídeos emissores de pósitron usados na medicina são produzidos por cíclotrons. A tabela 1 mostra os principais desses radionuclídeos e algumas de suas características físicas. Os radio- nuclídeos ${ }^{11} \mathrm{C}$ e ${ }^{15} \mathrm{O}$ são de elementos constituintes de organismos vivos, fato que os torna muito adequados para a marcação de biomoléculas. Por outro lado, como suas meias-vidas físicas são muito curtas, assim como a do ${ }^{13} \mathrm{~N}$, só podem ser utilizados se o acelerador para sua produção estiver nas dependências do próprio centro diagnóstico.

Hoje, o radionuclídeo mais usado é o ${ }^{18} \mathrm{~F}$, marcando a fluordeoxiglicose (FDG), um análogo da 
glicose que é consumido por células ativas, de tal maneira que sua presença indica função metabólica tecidual. Os quase 110 minutos de meia-vida do ${ }^{18} \mathrm{~F}$ permitem que a FDG marcada seja transportada a locais de exame razoavelmente afastados do centro de produção (em torno de $100 \mathrm{~km}$ por transporte terrestre), de modo que a PET realizada com $\left({ }^{18} \mathrm{~F}\right) \mathrm{FDG}$ é a dominante, com aplicações principalmente em oncologia e, em menor extensão, em neurologia, psiquiatria e cardiologia.

As imagens por emissão de pósitrons podem ser obtidas com dois tipos de equipamento: os sistemas dedicados e os baseados em câmaras de cintilação. Ambos utilizam a colimação eletrônica para registrar os eventos de coincidência, isto é, os pares de fótons que forem detectados em diferentes posições, dentro de um intervalo de tempo muito curto para caracterizar a coincidência, pré-definido pelo fabricante, vão constituir esses eventos. A linha que une os dois fótons detectados em coincidência define a linha de resposta, que é usada, posteriormente, na reconstrução do corte tomográfico. Se os dois fótons detectados provierem de uma mesma aniquilação, sem interagir com o meio, o evento é chamado de coincidência verdadeira, e o local de aniquilação estará sobre a linha de resposta. Se os fótons forem originados de uma mesma aniquilação, porém um deles tiver interagido com o meio, o local de aniquilação não estará mais sobre a linha de resposta e o evento é denominado espalhado. Se ambos os fótons se originarem de aniquilações diferentes, o par detectado definirá uma linha de resposta errada, resultando em um evento aleatório. A figura 2 ilustra esses eventos para um sistema dedicado, que é usado somente em estudos de PET.

Os modernos sistemas de PET dedicados são formados por mais de 15000 elementos de detecção, dispostos em anéis adjacentes, que vão registrar os eventos de coincidência dentro de intervalos da ordem de 10 a 12 nanossegundos. Os

FIGURA 2. Esquema de detecção por coincidência (pares de fótons) em sistemas dedicados de tomografia de emissão de pósitrons (PET) ${ }^{\mathrm{a}}$

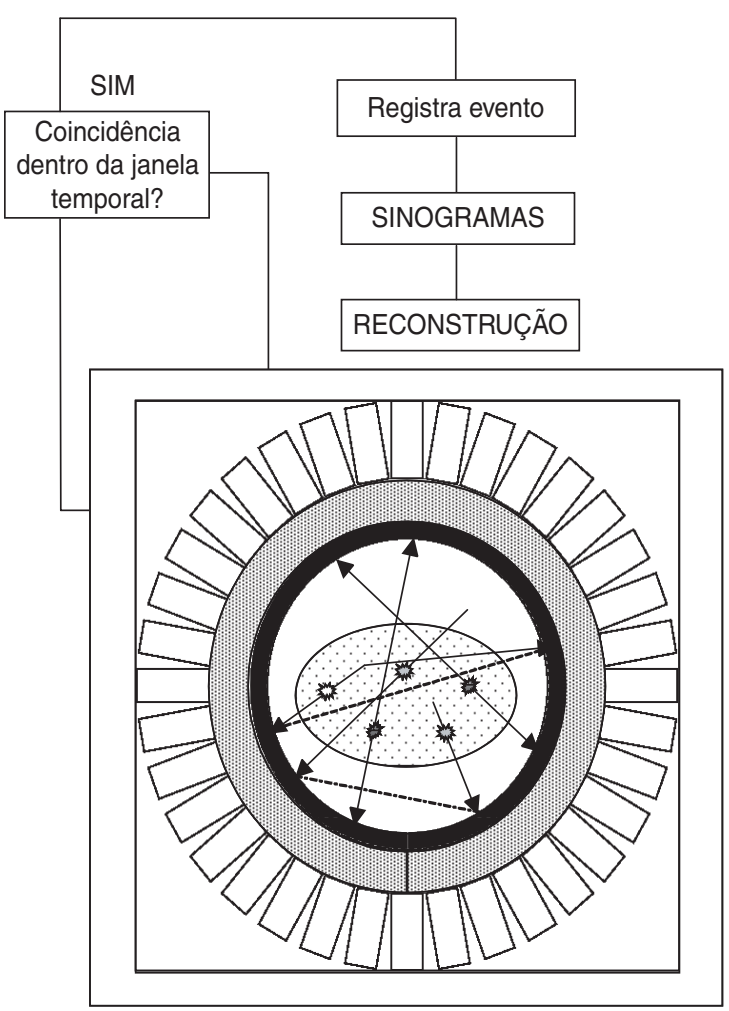

a Linhas de resposta cheias = eventos verdadeiros; linha de resposta tracejada = evento espalhado; linha de resposta traço-ponto $=$ evento aleatório. 
elementos de detecção são pequenos cristais de cintilação, BGO $\left(\mathrm{Bi}_{4} \mathrm{Ge}_{3} \mathrm{O}_{12}\right)$ ou LSO [ $\left(\mathrm{Lu}_{2} \mathrm{SiO}_{5}(\mathrm{Ce})\right]$, agrupados e acoplados a tubos fotomultiplicadores. As saídas dos tubos vão alimentar um sistema complexo de análise, discriminação e processamento que vai fornecer, no final, a imagem tomográfica. Como muitas aniquilações ocorrem simultaneamente nos volumes que contêm o radiofármaco, nem todos os eventos de coincidência registrados são formados por fótons criados na mesma aniquilação. Assim, é necessário excluir ou minimizar os eventos não-verdadeiros, para que a imagem reconstruída represente, da maneira mais próxima possível, a distribuição original.

Os sistemas baseados na câmara de cintilação são aqueles usados em SPECT dotados de circuitos de coincidência, isto é, a colimação eletrônica é instalada entre os dois detectores posicionados em oposição, permitindo o registro de eventos de coincidência e a posterior reconstrução de imagens por emissão de pósitrons. Assim, esse tipo de equipamento constitui uma alternativa ao custoso tomógrafo dedicado, principalmente quando a demanda não for suficiente para seu uso contínuo em PET. A grande diferença com relação ao tomógrafo dedicado está na menor eficiência de detecção dos fótons de $511 \mathrm{keV}$ pela câmara de cintilação. Mesmo assim, em diversas situações, os resultados obtidos com sistemas PET/SPECT fornecem informações clinicamente importantes.

Ambos os sistemas, dedicado ou não, permitem a aquisição de informações nos modos 2D e 3D. Os algoritmos de reconstrução mais utilizados são os iterativos e implementados em 2D. No caso de aquisição 3D, os dados registrados são reamostrados para que se possa aplicar a reconstrução 2D, que é menos custosa computacionalmente.

Várias correções são essenciais para se garantir a qualidade das imagens reconstruídas: de decaimento, devido à meia-vida física curta do ${ }^{18} \mathrm{~F}$; de atenuação e espalhamento, para reduzir os efeitos resultantes de interações dos fótons de $511 \mathrm{keV}$ com os tecidos; de eventos de coincidência aleatórios, que alocam erroneamente as origens das aniquilações; além de outras de menor impacto. Normalizações também devem ser realizadas para compensar a resposta não-uniforme do sistema de formação de imagens. Algumas dessas correções são implementadas no hardware, enquanto que outras são executadas via software, podendo ser incorporadas no próprio algoritmo de reconstrução.

É essencial que testes de calibração e controle de qualidade sejam executados periodicamente, para garantir a confiabilidade e a qualidade dos resultados, em especial se forem almejadas quantificações.

\section{A TOMOGRAFIA POR EMISSÃO DE PÓSITRONS NO BRASIL}

No Brasil, a tecnologia PET foi introduzida em 1998, com a instalação de uma câmara PET/ SPECT no Serviço de Radioisótopos do Instituto do Coração (InCor) do Hospital das Clínicas da Faculdade de Medicina da Universidade de São Paulo (HC-FMUSP). Essa nova tecnologia estendeu a metodologia já bem estabelecida em SPECT a PET a um custo reduzido e sustentável quando comparado ao custo da PET dedicada, além de permitir o uso contínuo da câmara quando da ausência de fornecimento da $\left({ }^{18} \mathrm{~F}\right) \mathrm{FDG}$. Posteriormente, outras cinco câmaras PET/SPECT foram instaladas na Cidade de São Paulo e uma na Cidade de Campinas, distante cerca de $100 \mathrm{~km}$. Dessas sete câmaras, três continuam produzindo imagens PET, enquanto que as outras são usadas somente em SPECT. Atualmente, mais dois sistemas desse tipo operam nas cidades do Rio de Janeiro e Brasília.

Os sistemas PET/SPECT familiarizaram a comunidade médica brasileira com a utilização de emissores de pósitrons, principalmente do ponto de vista dos protocolos clínicos, pois, até então, todos os estudos eram feitos com compostos emissores

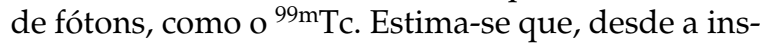
talação da primeira câmara PET/SPECT, cerca de 5000 exames foram realizados com esse tipo de equipamento em pacientes de todo o Brasil e de alguns países vizinhos.

No final de 2002, foi instalado o primeiro tomógrafo dedicado a PET no Serviço de Radioisótopos do InCor, substituindo o sistema PET/ SPECT. Até o início de 2004, outros três sistemas, do tipo combinado PET/CT, foram instalados na Cidade de São Paulo, todos em hospitais privados. A grande vantagem desses sistemas está na aquisição de duas modalidades de imagens a partir do mesmo referencial, isto é, o paciente não é deslocado entre um exame e outro, facilitando a fusão das duas imagens para a identificação das regiões analisadas. Devido à maior sensibilidade dos sistemas dedicados, é possível realizar uma quantidade maior de exames do que com os sistemas baseados em câmaras de cintilação, fato ilustrado pelos cerca de 2200 exames executados nos primeiros 18 meses da instalação dos sistemas dedicados.

Quanto à preparação dos pacientes, novos cuidados foram introduzidos, já que a FDG é consumida por tecidos metabolicamente ativos. Além disso, a manipulação de material com produção de fótons de aniquilação de $511 \mathrm{keV}$, bem maior do

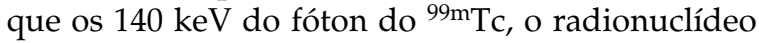
mais usado em medicina nuclear, exigiu uma nova 
FIGURA 3. Estudo de viabilidade do miocárdio com tomografias por emissão de pósitrons (PET) e de fótons simples $(\text { SPECT })^{\mathrm{a}}$
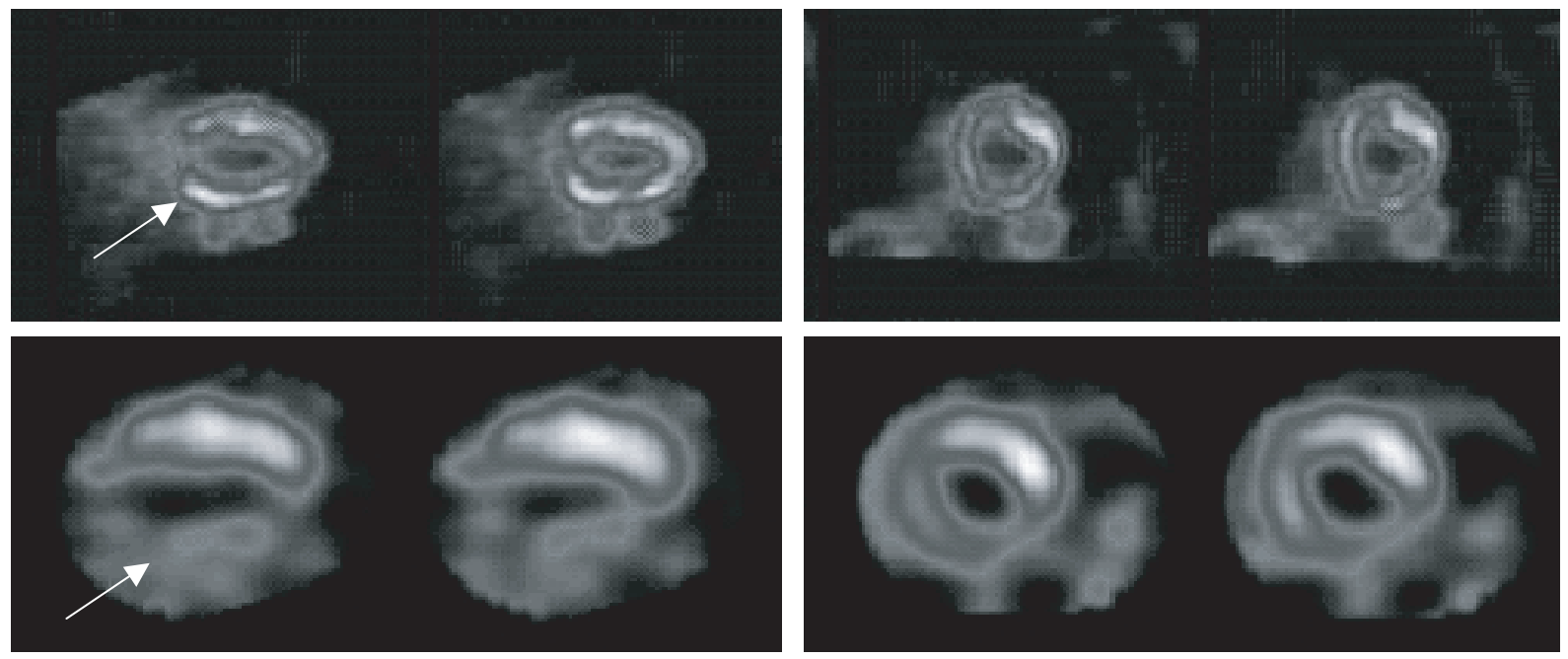

a Músculo viável: as imagens de metabolismo de glicose (PET), com FDG marcada com ${ }^{18} \mathrm{~F}$ (linha superior), mostram parede inferior ativa (indicada pela seta branca), enquanto que as imagens de perfusão sangüínea do miocárdio (SPECT), com tálio-201 (linha inferior, com fator de aumento maior que a linha superior), mostram a mesma parede hipoperfundida (indicada pela seta branca). As imagens foram gentilmente cedidas pelo Dr. J.C. Meneghetti, Serviço de Radioisótopos do Instituto do Coração, São Paulo (SP), Brasil.

FIGURA 4. Identificação a partir de tomografia por emissão de pósitrons (PET) de músculo não-viável para fins de revascularização do miocárdio ${ }^{\mathrm{a}}$
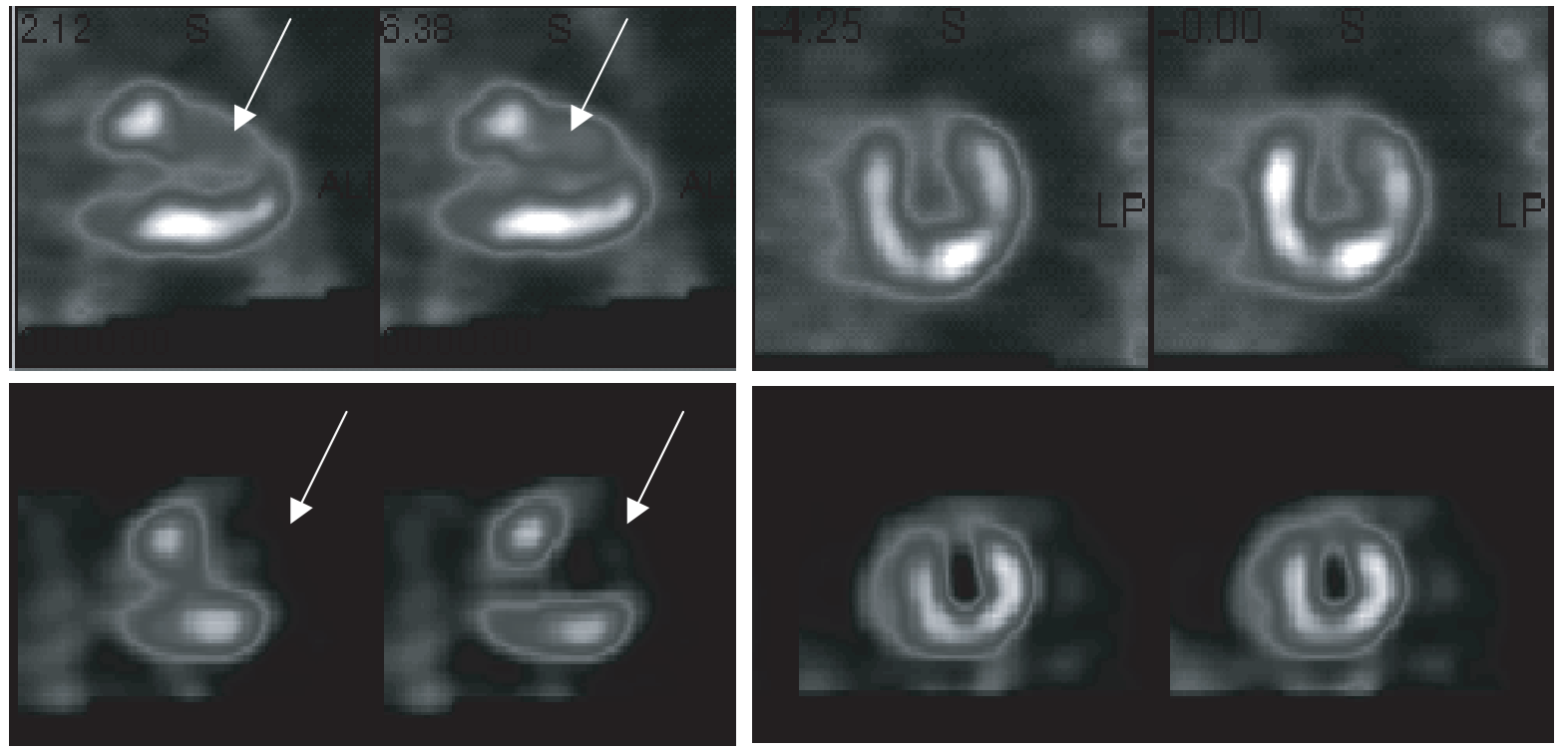

${ }^{a}$ As imagens de metabolismo de glicose (PET), com FDG marcada com ${ }^{18} \mathrm{~F}$ (linha superior), mostram parede anterior pouco ativa, enquanto que as imagens de perfusão

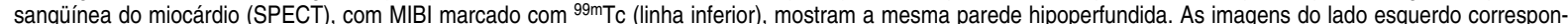
dem a cortes segundo o eixo cardíaco longo vertical, e as do lado direito, a cortes segundo o eixo cardíaco menor. A maior intensidade é representada por tonalidade mais clara, enquanto que a menor, por tonalidade mais escura. As imagens foram gentilmente cedidas pelo Dr. J.C. Meneghetti, Serviço de Radioisótopos do Instituto do Coração, São Paulo (SP), Brasil.

abordagem quanto à proteção radiológica. Por outro lado, a meia-vida física bastante curta facilitou o tratamento do rejeito.
A adoção de sistemas baseados em câmaras de cintilação também motivou os físicos-médicos que atuam em medicina nuclear a ampliar seus 
conhecimentos e adaptar procedimentos de controle de qualidade (24) e proteção radiológica, assim como desenvolver estudos e metodologias para a quantificação (25), reconstrução totalmente 3D (26) e fusão de imagens. A grande parte dos resultados conseguidos pode ser facilmente estendida aos sistemas dedicados.

Como no resto do mundo, a grande contribuição clínica dos estudos de PET com $\left({ }^{18}\right.$ F)FDG no Brasil está na oncologia, para detecção, localização e estadiamento de tumores primários, diferenciação entre tumores benignos e malignos, detecção e avaliação de recorrências e metástases, diferenciação entre recorrências e alterações pós-cirúrgicas, seguimento e avaliação de procedimentos terapéuticos. Os resultados obtidos, em especial aqueles com os sistemas combinados PET/CT, têm ajudado a indicar, ajustar e, até mesmo, alterar procedimentos em pacientes com tumores de diversos tipos.

A introdução da tomografia por emissão de pósitrons, em particular o uso de sistemas PET/ CT, está propiciando uma interação maior entre médicos nucleares e radiologistas no que se refere à análise e à avaliação das imagens compostas de anatomia e fisiologia, e entre os médicos especialistas em imagens e oncologistas no que tange aos resultados obtidos. Além disso, a possibilidade de utilização direta das informações metabólicas fornecidas pelas imagens de PET, combinadas com as informações anatômicas presentes na tomografia computadorizada por raios $X$, está também contribuindo para tornar o planejamento radioterapéutico mais adequado a cada paciente, principalmente quanto à proteção dos tecidos sãos ao redor do tumor.

Com relação às outras aplicações, o impacto tem sido menor, um pouco mais significativo em neurologia e psiquiatria do que em cardiologia, seguindo a distribuição das aplicações em países mais experientes em tecnologia PET. As figuras 3 e 4 ilustram dois estudos da viabilidade do miocárdio: um mostra um caso de músculo viável (ou hibernante), em que a intervenção com o objetivo de revascularização pode ser bem-sucedida, enquanto que o outro mostra que a atividade celular na região com hipoperfusão é muito baixa, indicando que o músculo é pouco viável.

Vale comentar que, segundo a legislação brasileira em vigor, a produção e a comercialização de radionuclídeos são exclusividade da Comissão Nacional de Energia Nuclear (CNEN). No momento, só duas instituições produzem o radiofármaco $\left({ }^{18} \mathrm{~F}\right)$ FDG no Brasil: o Instituto de Pesquisa em Energia Nuclear (IPEN/CNEN), em São Paulo (desde 1998), e o Instituto de Engenharia Nuclear (IEN/CNEN), no Rio de Janeiro (desde 2004) ${ }^{2}$. Esse fato limita a difusão dessa modalidade de imagem a outras regiões do território. Além disso, tal exclusividade é uma das duas causas para a tardia e lenta introdução da PET no cenário nacional. A outra razão é o alto custo da tecnologia e dos exames, que não são cobertos pelo sistema de saúde público. Dessa maneira, somente pacientes particulares e os que possuem planos de saúde que autorizam o reembolso têm acesso a essa tecnologia, que já é adotada há mais de uma década nos países desenvolvidos.

Atualmente, várias clínicas de São Paulo e Rio de Janeiro iniciaram o processo de compra de novos tomógrafos dedicados ou de sistemas PET/CT, pois a produção de $\left({ }^{18} \mathrm{~F}\right) \mathrm{FDG}$ nessas cidades já está em regime que permite um tal aumento. Além disso, a CNEN está considerando a instalação de mais dois cíclotrons, um na Região Nordeste e outro na Sudeste, com o objetivo de difundir e ampliar os estudos nessa área. Certamente, isso demandará um aumento de recursos humanos qualificados, tanto em termos de médicos especialistas como de físi-cosmédicos, radiofarmacêuticos, tecnólogos e pessoal de enfermagem, aspecto que deverá ser considerado pelas comunidades envolvidas em PET no país.

2 Em fevereiro de 2006, o Congresso Nacional Brasileiro promulgou uma emenda constitucional que flexibilizou a produção de radionuclídeos de meia-vida curta, tornando possível a produção de nuclídeos como o flúor-18, o carbono-11 e o oxigênio-15 por instalações que não estejam subordinadas à $\mathrm{CNEN}$, mas dentro das normas estabelecidas por ela. O número de equipamentos dedicados a estudos de PET, tomógrafos PET e PET/CT totaliza 12 em todo território nacional e tende a aumentar num futuro próximo, com a possibilidade de instalação de cíclotrons para a produção de FDG marcada com flúor-18.

\section{SYNOPSIS}

\section{Positron emission tomography: a new modality in Brazilian nuclear medicine}

In nuclear medicine, radioactive substances are used to diagnose and treat disease. This medical specialty, that can provide information about the human body's physiologic and metabolic processes, has become a key diagnostic tool for the early detection of many different disorders, including various types of cancer. The present article describes the historical milestones in nuclear medicine; the basic physical principles underlying positron emission tomography (PET), which is an imaging method used to map the distribution of radiopharmaceuticals in the body for diagnostic and therapeutic purposes, and the current status of this modality in Brazil.

Key words: radiopharmaceuticals, diagnostic imaging, PET, functional image, Brazil. 


\section{REFERÊNCIAS}

1. De Hevesy G. Radioelements as tracers in physics and chemistry. Chem News. 1913;108:166.

2. Blumgart HL, Weiss S. Studies on the velocity of blood flow. J Clin Invest. 1927;4(1):15-31.

3. Berson SA, Yalow RS, Bauman A, Rothschild MA, Newerly K. Insulin-I131 metabolism in human subjects: demonstration of insulin binding globulin in the circulation of insulin-treated subjects. J Clin Invest. 1956;35(2):170-90.

4. Lawrence EO, Livingstone MS. The production of high-speed light ions without the use of high voltage. Phys Rev. 1932; 40(1):19-30.

5. Cassen B, Curtis L, Reed C, Libby R. Instrumentation for ${ }^{131} \mathrm{I}$ use in medical studies. Nucleonics. 1951;9(2):46-50.

6. Anger HO. Scintillation camera. Rev Sci Instrum. 1958;29(1):27-33.

7. Harper PV, Beck R, Charleston D, Lathrop KA. Optimization of a scanning method using technetium-99m. Nucleonics. 1964;22(1):50-4.

8. Cherry SR, Sorenson JA, Phelps ME. Physics in nuclear medicine. $3^{a}$ ed. Filadélfia: WB Saunders; 2003.

9. Society of Nuclear Medicine. What is nuclear medicine? [folheto]. Disponível em http://interactive.snm.org/index. $\mathrm{cfm}$ ?PageID=3106\&RPID=972. Acessado em agosto de 2005.

10. Kuhl DE, Edwards RQ, Ricci AR, Yacob RJ, Mich TJ, Alavi A. The Mark IV system for radionuclide computed tomography of the brain. Radiology. 1976;121 (2):405-13.

11. Brownell GL, Burnham CA. MGH positron camera. NEREM Record. 1972; 2: 117.
12. Burnham CA, Brownell GL. A multicrystal positron camera. IEEE Trans Nucl Sci. 1972;NS-19(3):201-5.

13. Phelps ME, Hoffman EJ, Mullani NA Ter-Pogossian MM. Application of annihilation coincidence detection to transaxial reconstruction tomography. J Nucl Med. 1975;16(3):210-24.

14. Ter-Pogossian MM, Phelps ME, Hoffman EJ, Mullani NA. A positron emission transaxial tomograph for nuclear medicine imaging (PETT). Radiology. 1975;114(1):89-98

15. Chesler DA. Three-dimensional activity distribution from multiple positron scintigraphs. J Nucl Med. 1971;12(6): 347-8.

16. Sokoloff L, Reivich M, Kennedy C, Des Rosiers MH, Patlak CS, Pettigrew KD, et al. The $\left[{ }^{14} \mathrm{C}\right]$ deoxyglucose method for the measurement of local cerebral glucose utilization: theory, procedure and normal values in the conscious and anesthetized albino rat. J Neurochem. 1977;28(5):897-916.

17. Ido T, Wan C-N, Casella V, Fowler JS, Wolf AP, Reivich M, et al. Labeled 2deoxy-D-glucose analogs: ${ }^{18} \mathrm{~F}$ labeled 2-deoxy-2-fluoro-D-glucose, 2-deoxy-2fluoro-D-mannose and ${ }^{14} \mathrm{C}-2$-deoxy-2fluoro-D-glucose. J Label Compound Radiopharm. 1978;14:175-83.

18. Beyer T, Townsend DW, Brun T, Kinahan PE, Charron M, Roddy R, et al. A combined PET/CT scanner for clinical oncology. J Nucl Med. 2000; 41(8):1369-79.

19. Sweet WH. The use of nuclear disintegration in the diagnosis and treatment of brain tumor. N Engl J Med. 1951; 245(23):875-8

20. Wrenn FR, Good ML, Handler P. The use of positron-emitting radioisotopes for the localization of brain tumors. Science. 1951;113(1940):525-7.

21. Brownell GL, Sweet WH. Localization of brain tumors with positron emitters. Nucleonics. 1953;11(11):40-5.

22. Brownell GL. A history of positron imaging. Disponível em: http://www.mit. edu/ glb. Acessado em junho de 2005.

23. Ter-Pogossian MM, Powers WE. The use of radioactive oxygen- 15 in the determination of oxygen content in malignant neoplasms. Em: Radioisotopes in scientific research. Londres: Pergamon; 1958.

24. Aoki SM. Uma proposta para avaliação do desempenho de câmaras PET/ SPECT [dissertação de mestrado]. São Paulo: Instituto de Física da USP; 2002.

25. Pozzo L. Tomografia por emissão de pósitrons com sistemas PET/SPECT: um estudo da viabilidade de quantificação [tese de doutorado]. São Paulo: Instituto de Física da USP; 2005.

26. Palladino FH. Reconstrução 3D de imagens em tomografia por emissão de pósitrons com câmeras de cintilação [tese de doutorado]. São Paulo: Instituto de Física da USP; 2004.

Manuscrito recebido em 14 de dezembro de 2004 Aceito em versão revisada em 22 de abril de 2005. 